\title{
Low-dose versus Standard-Dose Leucovorin in the Treatment of Colon Cancer: Mansoura University Clinical Experience in Facing the Problem of Leucovorin Shortage
}

\author{
Hend M. H. R. Elkalla ${ }^{1}$, Mohamed S. Zahi ${ }^{1}$, Osama Eldamshety ${ }^{2}$ \\ ${ }^{1}$ Clinical Oncology and Nuclear Medicine Department, Mansoura University Hospitals, Faculty of \\ Medicine, Mansoura University, Mansoura, Egypt; ${ }^{2}$ Surgical Oncology, Oncology Center of \\ Mansoura University (OCMU), Faculty of Medicine, Mansoura University, Mansoura, Egypt
}

Background: Drug shortages have become a health care problem worldwide. One of the important drugs that are affected by shortage is leucovorin. It is used with 5-fluorouracil in many protocols for gastro-intestinal tumors and its shortage would have a negative impact on the treatment outcome.

Aim: To compare the effect of low-dose leucovorin versus the standard-dose in the treatment of stage II and III colon cancer.

Methods: The study included 100 patients with stage II or III adenocarcinoma of the colon who presented to the Clinical Oncology Department at Mansoura University Hospital from January 2011 till December 2012 after curative resection and were scheduled to receive adjuvant FOLFOX6 regimen. Patients were randomly allocated to receive standard-dose leucovorin $\left(200 \mathrm{mg} / \mathrm{m}^{2}\right.$, group 1) or low-dose leucovorin $\left(125 \mathrm{mg} / \mathrm{m}^{2}\right.$, group 2), both administered on days 1 and 2 over 2 hours infusion before 5-fluoruracil. The end points were toxicity, disease free survival (DFS) and overall survival (OS).

Results: The two study groups were matched regarding age, sex, and tumor stage. The mean DFS was 48.36 months for group 1 and 49.12 for group 2 with no significant difference $(\mathrm{p}=0.866)$. The mean OS was 65.07 months for group 1 and 62.45 for group 2 with no significant difference $(\mathrm{p}=0.544)$. Similarly, there was no significant difference between the two groups regarding hematosuppression, diarrhea, mucositis, neurotoxicity and the need for hospitalization.

Conclusion: Both protocols were well tolerated by all patients with no significant difference in the treatment outcome. The use of low-dose leucovorin protocol can help in solving the leucovorin shortage problem.

Keywords: Leucovorin, Drug shortage, Colon cancer, FOLFOX

Corresponding author: Hend M. H. R. Elkalla, MD; Clinical Oncology and Nuclear Medicine Department, Mansoura University Hospitals, Faculty of Medicine, Mansoura University, Mansoura, Egypt; Email: dr.hend1113@gmail.com

Submitted: 17-November-2018, Revised: 15-December-2018, Accepted: 21-December-2018, Published online: 2-January-2019

\section{INTRODUCTION}

Drug shortage problem has become a national health care disaster. It is an increasing crisis in the United States of America (USA) that adversely affects health care quality and causes elevation of health care costs ${ }^{1,2}$. The Food and Drug Administration (FDA) of the USA recorded 61 drug shortage incidents in 2005 which increased to 178 in $2010^{3,4}$.

Mostly, these shortages were for lowering the cost of non-original drugs or injectable drugs used in critical medicine fields, such as oncology and infectious diseases. The negative clinical effect of drug shortages occurs in the form of delays in medical steps causing medication mistakes, and drug-related side effects ${ }^{2,4}$.

Drug shortages are usually due to many causes. For example, if a generic drug is produced by few factories, and one of them has a production problem, the pressure on the other factories to supply enough drug amounts will increase with subsequent inadequate production capability which may cause primary shortage problem ${ }^{4}$, 5 . Contributing factors to drug shortages may include manufacturing quality aspects e.g. contamination, regularity issues, business decisions or disturbances within the supply chain ${ }^{1,2,6}$.

Shortage affected many drugs that play major roles in the treatment of adult and pediatric tumors, e.g. leucovorin, bleomycin, cisplatin, etoposide, vinblastine, vincristine, cytarabine, and methotrexate ${ }^{7,8}$. Alternatives for these drugs have been accompanied with an increase in the cost of health care ${ }^{8-10}$.

The increasing severity of the problem has warranted health care organizations to put a management strategy to prioritize treatment and also to provide substitutions to treatment plans. This includes clear decisions and steps for collecting data and decisionmaking ${ }^{1,11}$.

One of these important drugs is leucovorin. It is folic acid in a reduced form used in many chemotherapy protocols. Leucovorin is used with 5-fluorouracil in many protocols for gastrointestinal tumors and for many hematologic cancers as a rescue for high dose chemotherapy such as methotrexate ${ }^{1,12}$.

The FDA reported the $1^{\text {st }}$ leucovorin shortage problem in late 2008 that resulted from manufacturing 
delay by Bedford Laboratories which was the most leucovorin producing company worldwide and then by Teva Pharmaceuticals. This problem was ended by the spring of 2009. One year later, in 2010, the problem was repeated ${ }^{4}$. The shortage problem in 2010 was never resolved because both Bedford Laboratories and Teva Pharmaceuticals could not afford the need. The FDA reported in 2014 that the leucovorin shortage is still increasing ${ }^{13}$.

The tumor response rate in a study of 3300 patients with locally advanced colorectal cancer was assessed. Adding leucovorin to 5-fluorouracil increased the effect of 5-fluorouracil twice and the overall survival (OS) improved significantly ${ }^{12}$. Currently, the most accepted chemotherapeutic regimens in colorectal cancer includes the administration of leucovorin plus 5-fluorouracil alone or added to oxaliplatin as in FOLFOX or irinotecan as in FOLFIRI with or without monoclonal antibodies such as bevacizumab or cetuximab ${ }^{1}$.

In FOLFOX6 and FOLFIRI protocols, leucovorin $200 \mathrm{mg} / \mathrm{m}^{2}$ intravenous (IV) is taken on days 1 and 2 plus 5-fluorouracil. Due to the leucovorin shortage problem, health institutions employed alternative solutions, as removing or limiting the dose of leucovorin or shifting to levoleucovorin ${ }^{1}$.

We found that relatively few studies have assessed the clinical implications of leucovorin shortage in patients with colon cancer. In the current study, we compared the effect of low-dose leucovorin $\left(125 \mathrm{mg} / \mathrm{m}^{2}\right)$ to the standard-dose $\left(200 \mathrm{mg} / \mathrm{m}^{2}\right)$ on toxicity and survival in the adjuvant treatment setting of stage II and III colon cancer.

\section{METHODS}

This trial included 100 patients with colon cancer who presented to the Clinical Oncology and Nuclear Medicine Department at Mansoura University Hospital in the period from January 2011 to December 2012.

The inclusion criteria included: age $\geq 18$ years, stage II (pathologic staging T3N0 or T4N0 with high risk for systemic recurrence, poorly differentiated histology, lympho-vascular invasion, bowel obstruction or $<12$ lymph node examined) or III adenocarcinoma according to the American Joint Committee on Cancer (AJCC) staging, curative resection of the tumor, negative pregnancy test in females in the childbearing period and normal hematologic, hepatic and renal parameters. Patients were excluded from the trial if they had received chemotherapy before. Patients who received one dose of leucovorin IV as part of an adjuvant regimen containing fluorouracil for stage II or III colon cancer were included.

A central venous access devise was fixed before starting treatment. At the study beginning and before each cycle of chemotherapy, history, physical examination and complete blood count were performed. Toxicity was assessed using the common terminology criteria for adverse effects (CTCAE) version 4.0.

Two arms, randomized, parallel group design was used. Patients were randomly allocated to group 1 (control group) or group 2 (intervention group). Group 1 patients received 12 cycles of adjuvant FOLFOX6 protocol repeated every 2 weeks for six months (oxaliplatin $85 \mathrm{mg} / \mathrm{m}^{2}$ on day 1 over 90 minutes IV infusion + leucovorin $200 \mathrm{mg} / \mathrm{m}^{2}$ on days 1 and 2 over 2 hours IV infusion before fluorouracil + flurouracil 400 $\mathrm{mg} / \mathrm{m}^{2}$ IV bolus then $600 \mathrm{mg} / \mathrm{m}^{2}$ over 22 hours IV infusion on days 1 and 2). Group 2 patients received the same protocol with a reduction in the leucovorin dose to $125 \mathrm{mg} / \mathrm{m}^{2}$ administered on the same time and for the same infusion period on days 1 and 2 .

Patients were stratified by age $(<50$ years and $\geq 50$ years) and sex. The primary end points were toxicity, disease free survival (DFS) and OS.

\section{Statistical analysis}

We used SPSS version 22 for data analyses. Shapiro test was used firstly to test the normality of data.

Number and percent were used to describe qualitative data. Chi-square test was used to test the difference in proportions of categorical variables while Fischer exact test was used when an expected cell count was $<5$.

Continuous variables were presented as mean \pm standard deviation (SD) for parametric data and median for non-parametric data. The two groups were compared with Student $t$ test for parametric data and MannWhitney test for non-parametric data.

Survival analysis was tested by Kaplan-Meier test and the statistical significance of differences between curves was determined by Log-Rank test.

The threshold of significance is fixed at 5\% level (pvalue) for all the above mentioned statistical tests. If the probability of error is $<5 \%(\mathrm{p}<0.05)$ so it is significant. The smaller the $\mathrm{p}$-value obtained, the more significant are the results.

\section{RESULTS}

Patients' baseline characteristics are summarized in table 1 . Both groups were matched regarding age, sex and stage with no significant difference between them.

The overall follow up period ranged from 5 to 6 years. The median follow up period for group 1 was 62.5 months (range: 22-72) and for group 2 was 62.5 (range: 21-72) $(\mathrm{Z}=0.045, \mathrm{p}=0.964)$. The treatment outcome of both groups is summarized in table 2. There was no significant difference between the two groups as regards outcome. The relapse rate was $38 \%$ and $36 \%$ in the treatment groups respectively.

None of the assessed toxicities differed significantly between the two treatment groups as well as the need for hospitalization (table 3 ).

At the time of analysis, the median DFS and OS were not reached for both treatment groups. The estimated means of DFS and OS in both groups are shown in table 4 . There was no significant difference between the two groups as regards DFS and OS. Figures 1 and 2 show the DFS and OS curves. 
Table 1: Patients' characteristics

\begin{tabular}{|c|c|c|c|c|}
\hline Variables & Group $1(n=50)$ & Group $2(n=50)$ & Test of significance & p-value \\
\hline \multicolumn{5}{|l|}{ Age/years } \\
\hline Mean \pm SD & $49.08 \pm 6.99$ & $48.84 \pm 6.60$ & $\mathrm{t}=0.176$ & 0.860 \\
\hline Min-Max & $37-61$ & $38-62$ & & \\
\hline \multicolumn{5}{|l|}{ Sex } \\
\hline Male & $26(52 \%)$ & $27(54 \%)$ & $\chi^{2}=0.04$ & 0.841 \\
\hline Female & $24(48 \%)$ & $23(46 \%)$ & & \\
\hline \multicolumn{5}{|l|}{ Stage } \\
\hline II & $25(50 \%)$ & $24(48 \%)$ & $\chi^{2}=0.04$ & 0.841 \\
\hline III & $25(50 \%)$ & $26(52 \%)$ & & \\
\hline
\end{tabular}

Group 1: Standard-dose leucovorin, Group 2: Low-dose leucovorin, $t$ : student t-test, $\chi^{2}$ : chi square test

Table 2: Treatment outcome

\begin{tabular}{|c|c|c|c|c|}
\hline Variables & Group $1(n=50)$ & Group $2(n=50)$ & Test of significance & p-value \\
\hline \multicolumn{5}{|l|}{ Relapse (metastasis or recurrence) } \\
\hline Yes & $19(38 \%)$ & $18(36 \%)$ & \multirow{2}{*}{$\chi^{2}=0.043$} & \multirow[t]{2}{*}{0.836} \\
\hline No & $31(62 \%)$ & $32(64 \%)$ & & \\
\hline \multicolumn{5}{|l|}{ Time to recurrence (months) } \\
\hline Median (Min-Max) & $56(6-66)$ & $56(9-66)$ & $\mathrm{Z}=0.135$ & 0.893 \\
\hline \multicolumn{5}{|l|}{ Status at last assessment } \\
\hline Alive on regular follow up & $33(66 \%)$ & $34(68 \%)$ & \multirow[t]{3}{*}{$\chi^{2}=0.355$} & \multirow[t]{3}{*}{0.551} \\
\hline Died & $7(14 \%)$ & $10(20 \%)$ & & \\
\hline Lost to follow up & $10(20 \%)$ & $6(12 \%)$ & & \\
\hline
\end{tabular}

Group 1: Standard-dose leucovorin, Group 2: Low-dose leucovorin, $\chi^{2}$ : chi square test, Z: Mann Whitney test

Table 3: Side effects and the need for hospitalization

\begin{tabular}{|c|c|c|c|c|}
\hline \multirow[t]{2}{*}{ Variables } & Group $1(n=50)$ & Group $2(n=50)$ & \multirow[t]{2}{*}{$\chi^{2}$} & \multirow[t]{2}{*}{ p-value } \\
\hline & n (\%) & n (\%) & & \\
\hline Haemato-suppression & $22(44)$ & $23(46)$ & 0.04 & 0.84 \\
\hline Diarrhea & $12(24)$ & $11(22)$ & 0.05 & 0.812 \\
\hline Mucositis & $8(16)$ & $7(14)$ & 0.08 & 0.779 \\
\hline Neurotoxicity & $11(22)$ & $12(24)$ & 0.05 & 0.812 \\
\hline Hospitalization & $4(8)$ & $3(6)$ & FET & 1 \\
\hline
\end{tabular}

Group 1: Standard-dose leucovorin, Group 2: Low-dose leucovorin, FET: Fischer exact test

Table 4: Kaplan Meier survival analysis

\begin{tabular}{|c|c|c|c|c|c|}
\hline & Overall & Group 1 & Group 2 & Log Rank & p value \\
\hline \multicolumn{6}{|l|}{ Disease-free survival } \\
\hline $\begin{array}{l}\text { Estimated mean } \\
(95 \% \mathrm{CI})\end{array}$ & $\begin{array}{l}48.74 \\
(44.254-53.226)\end{array}$ & $\begin{array}{l}48.36 \\
(42.004-54.716)\end{array}$ & $\begin{array}{l}49.12 \\
(42.791-55.449)\end{array}$ & 0.028 & 0.866 \\
\hline $\begin{array}{l}\text { Estimated median } \\
(95 \% \mathrm{CI})\end{array}$ & Not reached & Not reached & Not reached & & \\
\hline \multicolumn{6}{|l|}{ Overall survival } \\
\hline $\begin{array}{l}\text { Estimated mean } \\
(95 \% \mathrm{CI})\end{array}$ & $\begin{array}{l}63.702 \\
(60.118-67.287)\end{array}$ & $\begin{array}{l}65.075 \\
(60.286-69.864)\end{array}$ & $\begin{array}{l}62.455 \\
(57.201-67.708)\end{array}$ & 0.369 & 0.544 \\
\hline $\begin{array}{l}\text { Estimated median } \\
(95 \% \mathrm{CI})\end{array}$ & Not reached & Not reached & Not reached & & \\
\hline
\end{tabular}

Group 1: Standard-dose leucovorin, Group 2: Low-dose leucovorin, CI: Confidence interval

\section{DISCUSSION}

Leucovorin, which is a cornerstone in chemotherapeutic protocols used in treatment of colorectal cancer, is one of the common drugs that faces shortage.

Our work is one of the few trials exploring a strategy to overcome leucovorin shortage in patients with colon cancer. The study included two homogenous groups of colon cancer patients with stage II or III disease and good matched age and sex. Generally, in this study, the new strategy of reduced leucovorin dosage was well tolerated by our patients with no negative impact on the clinical outcome. There were no differences in DFS or OS between the two studied groups.

We found difficulty in comparing our work to the work of others. Most of the studies that investigated the shortage of leucovorin focused on its financial impact on health care systems. In Jager et al study, which was reported in 1996, there was no difference in response rate or survival in patients suffering from metastatic colorectal who received low-dose leucovorin $\left(50 \mathrm{mg} / \mathrm{m}^{2}\right)$ plus bolus fluoruracil when compared to those receiving high dose leucovorin $\left(500 \mathrm{mg} / \mathrm{m}^{2}\right)^{14}$. This agrees with the principle of our study. 


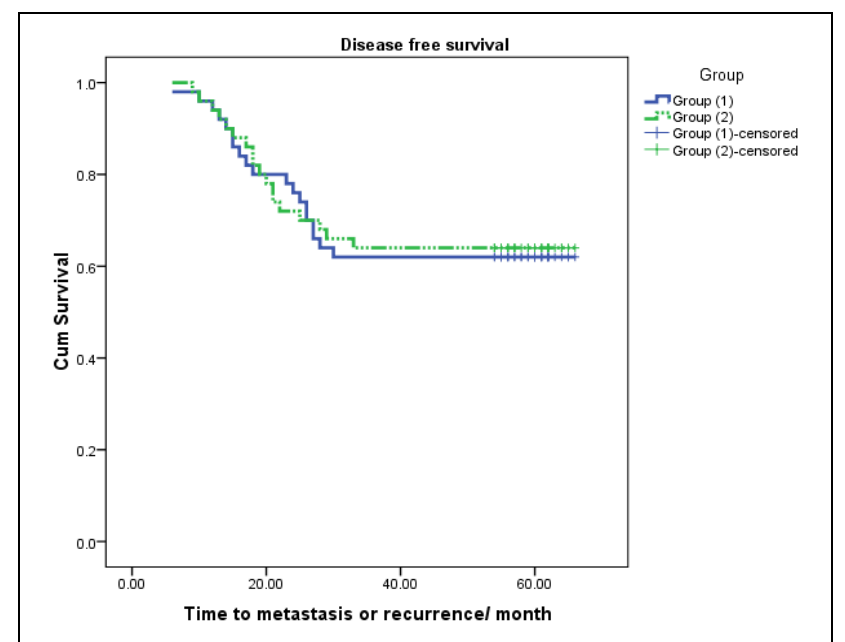

Figure 1: Kaplan Meier disease free survival curves (Group 1: Standard-dose leucovorin, Group 2: Low-dose leucovorin)

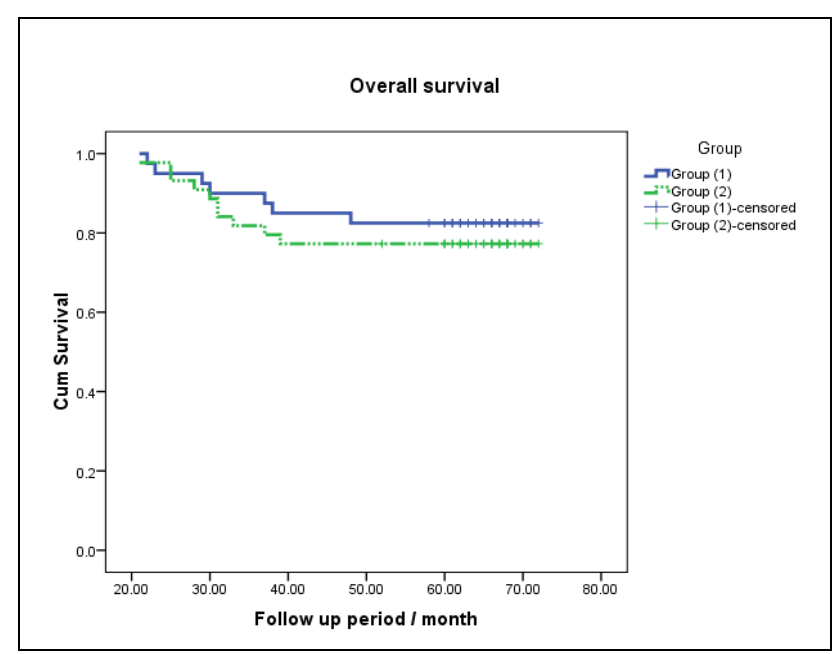

Figure 2: Kaplan Meier overall survival curves (Group 1: Standard-dose leucovorin, Group 2: Low-dose leucovorin)

The QUASAR study found that the usage of lowdose leucovorin of $25 \mathrm{mg}$ was associated with the same survival and 3 years recurrence rate as the dose of 175 mg taken with bolus 5-flourouracil in the adjuvant therapy setting for colorectal cancer ${ }^{15}$. Our results matched those of that study and confirm that there is no difference between standard and low-dose of leucovorin in the adjuvant setting of colon cancer.

In 2013, Hanna et al reviewed the medical records of outpatients treated with leucovorin at the Continuum Cancer Centers of New York between April and September of 2010, 2011, and 2012. They reported the pattern of leucovorin use (dose and number of cycles) and the change in this pattern over years. The administered leucovorin dose was either low-dose (20$\left.40 \mathrm{mg} / \mathrm{m}^{2}\right)$ or high dose $\left(200-500 \mathrm{mg} / \mathrm{m}^{2}\right)$ They found that low-dose leucovorin was used in $30 \%$ of doses in 2010, 30.4\% in 2011 and $99.1 \%$ in 2012 ( $p<0.0001$ ). Patient characteristics and stage were comparable between years $(p=0.87)$. Among patients treated for colon cancer; they found no relation between stage (III or IV) and use of low-dose leucovorin in 2010 or 2011 16 .

Recently, Reynolds et al reported the results of a study addressing the same point of research. They used smaller dose of leucovorin $20 \mathrm{mg} / \mathrm{m}^{2}$ in the FOLFOX6 protocol for metastatic colorectal cancer based on the results of prospective trials suggesting that smaller dose of leucovorin may have the same effectiveness in other protocols containing fluorouracil. They found no difference in OS of metastatic colorectal cancer cases treated with $1^{\text {st }}$ line FOLFOX6 with low- vs. high-dose leucovorin. Although they studied low-dose leucovorin FOLFOX6 regimen in the metastatic setting, their results confirm ours in terms of the clinical efficacy of low-dose leucovorin containing regimens ${ }^{17}$.

Shank et al studied adult patients receiving leucovorin as a part of protocols containing fluorouracil. The study included patients with stage II or III colorectal cancer and those with newly diagnosed metastatic disease. One low fixed dose of leucovorin $(50 \mathrm{mg})$ was used and compared to one body surface area adjusted dose $\left(200-500 \mathrm{mg} / \mathrm{m}^{2} / \mathrm{dose}\right)$. They concluded that there is no significant difference in progression free survival $(p=0.254)$, OS $(p=0.923)$, or side effects; which is similar to our results. However, it should be noted that they included metastatic disease in addition to stage II and III in their study ${ }^{1}$.

The proposed low-dose leucovorin in our study is one of the ideal solutions to standardize dosing with possible decline in mistakes that may result during dose calculation. In addition to decreasing waste and making treatment protocols much simple and easier. Our results may help with further studies recruiting larger patient number to resolve the problem of leucovorin shortage and to minimize its cost that overburden our institute financially.

\section{Conclusion}

Our trial demonstrated that low-dose leucovorin is equally effective to regular dose leucovorin with no negative impact on DFS or OS in patients with colon cancer treated by adjuvant chemotherapy. The use of low-dose leucovorin protocol can help in solving the leucovorin shortage problems in the near future.

\section{Conflict of interest}

The authors have no conflict of interest to declare.

\section{Funding}

None.

\section{REFERENCES}

1- Shank BR, Seung AH, Kinsman K, Newman MJ, Donehower RC, Burton B. Effects of the leucovorin shortage: Pilot study investigating cost, efficacy, and toxicity comparison of low fixed-dose versus body surface area-adjusted leucovorin dosing in patients with resectable colon or metastatic colorectal cancer. J Oncol Pharm Pract. 2017; 23(3): 163-172.

2- Stein R. Shortages of key drugs endanger patients. The Washington Post, 2011.

3- Food and Drug Administration. FDA works to lessen drug shortage impact. Available from https://www.fda.gov/forconsumers/consumerupdates/ucm 258152.htm . Accessed: 27-December-2018.

4- Hayes MS, Ward MA, Slabaugh SL, Xu Y. Lessons from the leucovorin shortages between 2009 and 2012 in a 
medicare advantage population: where do we go from here? Am Health Drug Benefits. 2014; 7(5): 264-270.

5- American Society of Health-System Pharmacists. Drug Shortages. Available from https://www.ashp.org/DrugShortages. Accessed: 27-December-2018.

6- Fox ER, McLaughlin MM. ASHP guidelines on managing drug product shortages. Am J Health Syst Pharm. 2018; 75(21): 1742-1750.

7- Kaiser J. Medicine. Shortages of cancer drugs put patients, trials at risk. Science. 2011; 332(6029): 523.

8- Link MP, Hagerty K, Kantarjian HM. Chemotherapy drug shortages in the United States: genesis and potential solutions. J Clin Oncol. 2012; 30(7): 692-694.

9- Kehl KL, Gray SW, Kim B, et al. Oncologists' experiences with drug shortages. J Oncol Pract. 2015; 11(2): e154-162.

10- Gatesman ML, Smith TJ. The shortage of essential chemotherapy drugs in the United States. N Engl J Med. 2011; 365(18): 1653-1655.

11- Ventola CL. The drug shortage crisis in the United States: causes, impact, and management strategies. P T. 2011; 36(11): 740-757.

12- Thirion P, Michiels S, Pignon JP, et al. Modulation of fluorouracil by leucovorin in patients with advanced colorectal cancer: an updated meta-analysis. J Clin Oncol. 2004; 22(18): 3766-3775.
13- U.S. Food and Drug Administration. Current and Resolved Drug Shortages and Discontinuations Reported to FDA: Leucovorin Calcium Lyophilized Powder for Injection. Available from: https://www.accessdata.fda.gov/scripts/drugshortages/dsp ActiveIngredientDetails.cfm?AI=Leucovorin+Calcium+ Lyophilized+Powder+for+Injection\&st=c\&tab=tabs4\&panels $=1$. Accessed 27-December-2018.

14- Jäger E, Heike M, Bernhard $\mathrm{H}$, et al. Weekly high-dose leucovorin versus low-dose leucovorin combined with fluorouracil in advanced colorectal cancer: results of a randomized multicenter trial. Study Group for Palliative Treatment of Metastatic Colorectal Cancer Study Protocol 1. J Clin Oncol. 1996; 14(8): 2274-2279.

15- Quasar Collaborative Group, Gray R, Barnwell J, et al. Adjuvant chemotherapy versus observation in patients with colorectal cancer: a randomised study. Lancet. 2007; 370 (9604): 2020-2029.

16- Hanna MSE, Kozuch P, Thorn M, el al. Impact of leucovorin shortage on patient treatment. J Clin Oncol. 2013; 31(15 suppl): e17566.

17- Reynolds J, Chamberland-Tremblay A, Herrington JD, Munoz Maldonado Y, Wong L. High- versus low-dose leucovorin in the modified FOLFOX6 regimen for firstline treatment of metastatic colorectal cancer. J Oncol Pharm Pract. 2017; 23(3): 173-178. 